\title{
FBK-HLT-NLP at SemEval-2016 Task 2: A Multitask, Deep Learning Approach for Interpretable Semantic Textual Similarity
}

\author{
Simone Magnolini \\ Fondazione Bruno Kessler \\ University of Brescia \\ Brescia, Italy \\ magnolini@fbk.eu
}

\author{
Anna Feltracco \\ Fondazione Bruno Kessler \\ University of Pavia \\ Pavia, Italy \\ feltracco@fbk.eu
}

\author{
Bernardo Magnini \\ Fondazione Bruno Kessler \\ Povo-Trento, Italy \\ magnini@fbk.eu
}

\begin{abstract}
We present the system developed at FBK for the SemEval 2016 Shared Task 2 "Interpretable Semantic Textual Similarity" as well as the results of the submitted runs. We use a single neural network classification model for predicting the alignment at chunk level, the relation type of the alignment and the similarity scores. Our best run was ranked as first in one the subtracks (i.e. raw input data, Student Answers), among 12 runs submitted, and the approach proved to be very robust across the different datasets.
\end{abstract}

\section{Introduction}

The Semantic Textual Similarity (STS) task measures the degree of equivalence between the meaning of two texts, usually sentences. In the Interpretable STS (iSTS) (Agirre et al., 2016) the similarity is calculated at chunk level, and systems are asked to provide the type of the relationship between two chunks, as an interpretation of the similarity. Given an input pair of sentences, participant systems were asked to: (i) identify the chunks in each sentence; (ii) align chunks across the two sentences; (iii) indicate the relation between the aligned chunks and (iv) specify the similarity score of each alignment.

The iSTS task has already been the object of an evaluation campaign in 2015, as a subtask of the SemEval-2015 Task 2: Semantic Textual Similarity (Agirre et al., 2015). More in general, shared tasks for the identification and measurement of STS were organized in 2012 (Agirre et al., 2012), 2013 (Agirre et al., 2013) and 2014 (Agirre et al., 2014).
Data provided to participants include three datasets: image captions (Images), pairs of sentences from news headlines (Headlines), and a question-answer dataset collected and annotated during the evaluation of the BEETLE II tutorial dialogue system (Student Answers) (Agirre et al., 2015). For each dataset, two subtracks were released: the first with raw input data (SYS), the second with data split in gold standard chunks (GS). Given these input data, participants were required to identify the chunks in each sentence (for the first subtrack only), align chunks across the two sentences, specify the semantic relation of the alignment - selecting one of the following: EQUI for equivalent, OPPO for opposite, SPE1 and SPE2 if chunk in sentence 1 is more specific than chunk in sentence 2 and vice versa, SIMI for similar meanings, REL for chunks that have related meanings, and NOALI for chunk has no corresponding chunk in the other sentence (Agirre et al., 2015)-, and provide a similarity score for each alignment, from 5 (maximum similarity/relatedness) to 0 (no relation at all). In addition, an optional tag for alignments showing factuality (FACT) or polarity (POL) phenomena, can be specified. The evaluation is based on (Melamed, 1998), which uses the F1 of precision and recall of token alignments.

We participate in the iSTS shared task with a system that combines different features - including word embedding and chunk similarity - using a Multilayer Perceptrons (MLP). Our main contribution was focused on the optimization of a Neural Network setting (i.e. topology, activation function, multi-task training) for the iSTS task. We show that 
even with a relatively small and unbalanced training dataset, a neural network classifier can be built that achieves results very close to the best system. Particularly, our system makes use of a single model for the different training sets of the task, proving to be very robust to domain differences.

The paper is organized as follows. Section 2 presents the system we built; Section 3 reports the results we obtained and an evaluation of our system. Finally, Section 4 provides some conclusions.

\section{System Description}

Our system is built combining different linguistic features in a classification model for predicting chunk-to-chunk alignment, relation type and STS score. We decide to use the same features for all these three subtasks and to use a unique multitask MLP with shared layers for all the subtasks. The system is expandable and scalable for adopting more useful features aiming at improving the accuracy.

In this Section, we describe the pre-processing of the data, the features we used, the MLP structure, its training, its output and, finally, the difference between the three submitted runs.

\subsection{Data Pre-processing}

The input data undergo a data pre-processing in which we use a Python implementation of MBSP (Daelemans and Van den Bosch, 2005), a library providing tools for tokenization, sentence splitting, part of speech tagging, chunking, lemmatization and prepositional phrase attachment. The MBSP chunker is used in the SYS subtrack, which requires participants to identify the chunks in each sentence. For both subtracks, we pre-processed the initial datasets of sentence pairs by pairing all the chunks in the first sentence with all the chunks in the second sentence. Henceforth, we will refer to the two chunks in each of the obtained pairs as chunk1 and chunk2, being chunk1 a chunk of the first sentence and chunk2 a chunk of the second sentence.

\subsection{Feature Selection}

To compute the chunk-to-chunk alignment, the relation type and the STS score we use a total of 245 features.
Chunk tags. A total of 18 features ( 9 for chunkl and 9 for chunk2) are related to chunk tags (e.g. noun phrase, prepositional phrase, verb phrase).

For each chunk in the SYS datasets -chunked with MBSP- the system takes into consideration the chunk tags as identified by that library. ${ }^{1}$

For the GS datasets -already chunked datasets- the system first re-chunks the datasets with MBSP and than evaluates if chunks in the GS corresponds to chunks as identified in MBSP. If this is the case, chunk tag is extracted; otherwise the systems does the same operation (i.e. re-chunking and tag extraction) using pattern.en (De Smedt and Daelemans, 2012), a regular expressions-based shallow parser for English that uses a part-of-speech tagger extended with a tokenizer, lemmatizer and chunker. ${ }^{2}$

If no corresponding chunk is found, no chunk tag is assigned.

Token and lemma overlap. Four further features are related to tokens and lemmas overlap between a pair of chunks. In particular, the system considers the percentage of (i) tokens and (ii) lemmas in chunks 1 that are present also in chunk2 and viceversa (iii - iv).

WordNet based features. Another group of features concerns lexical and semantic relations between words extracted from WordNet 3.0 (Fellbaum, 1998). As such, we evaluate the type of relation between chunks by considering all the lemmas in the two chunks and checking whether a lemma in chunkl is a synonym, antonym, hyponym, hyperonym, meronym or holonym of a lemma in chunk2. The relations between all the combinations of the lemmas in the two chunks are extracted. The presence or absence of a relation is consider a feature at chunk level (for a total of 6 features for chunk1 and 6 features for chunk2).

Furthermore, we consider as a feature the synset similarity existing in the WordNet hierarchy between each lemma in the two chunks, as calculated

\footnotetext{
${ }^{1}$ The chunk tags are the following: noun phrase (NP), prepositional phrase (PP), verb phrase (VP), adverb phrase (ADVP), adjective phrase (ADJP), subordinating conjunction (SBAR), particle (PRT), interjection (INTJ), prepositional noun phrase (PNP).

${ }^{2}$ The two chunkers use the same set of chunk tags.
} 
by pattern.en. We calculate the average of the best alignments for each lemma in the two chunks. For example, consider the chunk pair: chunk1 "the animal" and chunk2 "the sweet dog". For each lemma in chunkl, for which a synset can be retrieved from WordNet, ("animal"), we calculate the maximum similarity with lemmas in chunk2. Thus, for this pair of chunks the resulting maximum similarity is between "animal-dog" $=0.299$ (being equal to 0.264 for "animal-sweet"). The chunk similarity score is 0.299 . With the same strategy we calculate similarity between lemmas in chunk2 towards chunkl, i.e. "sweet-animal" $=0.264$, "doganimal" $=0.299$ resulting in a chunk similarity score of $[(0.264+0.299) / 2]=0.281$. If lemmas were not found in WordNet, the synset similarity is considered 0 .

Word embedding. We use a distributional representation of the chunk for a total of 200 features (100 for chunk1 and 100 for chunk2) by first calculating word embedding and then combining the vectors of the words in the chunk (i.e. by calculating the element wise mean of each vector). We use Mikolov word2vec (Mikolov et al., 2013) with 100 dimensions using ukWaC, GigaWords (NYT), Europarl V.7, Training Set (JRC) corpora.

The system computes the chunk-to-chunk similarity by calculating the cosine similarity between the two chunk vectors with three different models: the first uses the already described vectors (one feature); the second uses vectors representations extracted with a different corpus and a different parametres -i.e. Google News, with 300 dimensions of the vectors- (one feature); the third uses GloVe vectors (Pennington et al., 2014) with 300 dimensions (one feature).

Baseline feature. The baseline output - provided by the organizers (Agirre et al., 2016) - was also exploited, i.e. we consider if the chunks are evaluated as aligned, if chunkl is not aligned, if chunk2 is not aligned (3 features).

Composition of the input data. The last three features refer to the datasets. The system takes into consideration if the chunks are extracted from Headline, Images, or Student Answers dataset.

\begin{tabular}{lr}
\hline & \#features \\
\cline { 2 - 2 } Chunk tags & 18 \\
Token and lemma overlap & 4 \\
WordNet relations and similarity & 14 \\
Word embedding & 200 \\
Cosine Similarity & 3 \\
Baseline feature & 3 \\
Composition of the input data & 3 \\
\cline { 2 - 2 } Total & 245 \\
\hline
\end{tabular}

Table 1: Feature Selection.

\subsection{Neural Network}

We use a multitask MLP (see Figure 1) to classify chunk pairs, implemented with the TensorFlow library (Abadi et al., 2015). The system uses three classifiers: one for the chunk alignment, one for alignment type, one for STS score. The input layer has 245 entities, so we use fully connected hidden layers with 250 nodes. During the test we observed that smaller (200 nodes) or bigger (300 nodes) layers reduce the performances. The system is composed by two layers (i.e. L1 and L2) shared between the three classifiers. On the top of them there are other two layers: the former (L3a) used only for the alignment classifier and the latter (L3b) shared among the score classifier and the type classifier. At the very end of L3b, there are other two layers one for the score (L4a) and one for the type (L4b). In synthesis for alignment there are three hidden layers, two shared (L1 and L2) and one private (L3a), for STS score there are four hidden layers, three shared (L1, L2, L3b) and one private (L4a) and the same for the type labeling (L1, L2, L3b + L4b). Every output layers is a softmax; during the training the system has a dropout layer that remove nodes from the layer with a probability of $50 \%$ to avoid overfitting.

We use sigmoid as activation function as it results the best one during the development test among all the activation function available in the library. Finally, we train our MLP using three different optimizers; each of them reduces the softmax error on a subtasks (i.e. alignment, type labeling or STS score). For the optimization we use the Adam algorithm (Kingma and $\mathrm{Ba}, 2014$ ) with different learning rates: 0.00006 for the first classifier and 0.00004 for 


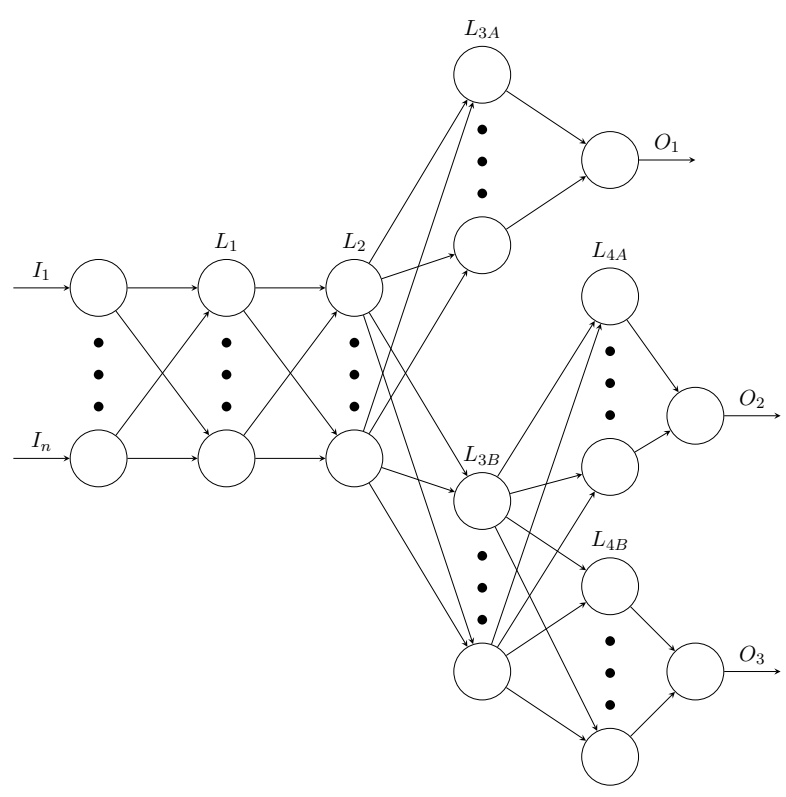

Figure 1: Multitask learning Neural Network.

the other ones.

We train the classifiers for three cycles. This training strategy is driven by learning curves analysis: we keep training the classifiers until the learning curves keep growing. We notice that the alignment classifier stops learning earlier, followed by the relation type classifier, and, at the end, the STS score classifier. Under these findings, to train all the classifiers in the same way overfits the training data. Furthermore, the training data are very unbalanced (most of the pairs are not aligned); thus, we use random minibatches with a fix proportion between aligned pairs and unaligned pairs. To do so, we use the unaligned pairs more than once in a single training epoch. In particular, first we train the alignment classifier with the following proportion: $2 / 5$ of aligned examples and $3 / 5$ of not aligned pairs, for 8 training epochs (i.e. every aligned pair is used as training data at least 8 times). The second training cycle optimizes relation type labeling and STS score, with the proportion of 9/10 aligned and 1/10 not aligned for other 8 training epochs. Finally, in the third training cyle, we train only for STS score with a proportion of 9/10 aligned and 1/10 not aligned pairs.

\subsection{Output}

We combine the output of the three classifiers (alignment, relation type and similarity score) organized in a pipeline. First, we label as "not aligned" all the punctuation chunks (i.e. those defined as "not alignable" by the baseline); then we label as "aligned" all the chunks aligned by the first classifier, allowing multiple alignments for each chunk. For every aligned chunk pair we add the type label and the STS score. We do not take into consideration chunk pairs classified as "not aligned" by the first classifier even if they are classified with a label different from NOTALI or with an STS score higher than 0 .

\subsection{Submitted Runs}

We submitted three runs, with different training settings. In the first run we use all the training data with a mini-batch of 150 elements. In the second run we train and evaluate separately each dataset with a mini-batch of 150 elements. Finally, in the third run we use all the training data with a mini-batch of 200 elements. We choose these settings in order to evaluate how in-domain data and different sizes of the mini-batch influence the classification results.

\section{Results and Evaluation}

Table 2 compares the results of our runs with the baseline and the best system for each subtrack of the three datasets, showing:

- F1 on alignment classification (F);

- F1 on alignment classification plus relation alignment type (+T);

- F1 on alignment classification plus STS score $(+\mathrm{S})$;

- F1 on alignment classification plus relation alignment type and STS score (+TS);

- Ranked position over the runs submitted: i.e. 13 runs for Images and Headlines SYS, 12 for Student Answer SYS, 20 for Images and Headlines GS and 19 for Student Answer GS (RANK)

Table 2 shows that for all the six subtracks run 1 and run 3 register better results. In particular, for what concerns GS subtasks (with already chunked sentences), run2 is ranked at least two positions lower with respect to the other two runs. Since the difference between run 2 and the other runs lays on the data used for training, these results seem to 


\begin{tabular}{|c|c|c|c|c|c|c|c|c|c|c|}
\hline & \multicolumn{5}{|c|}{ IMAGES SYS } & \multicolumn{5}{|c|}{ IMAGE GS } \\
\hline & $\mathrm{F}$ & $+\mathrm{T}$ & $+\mathrm{S}$ & $+\mathrm{TS}$ & RANK & $\mathrm{F}$ & $+\mathrm{T}$ & $+\mathrm{S}$ & $+\mathrm{TS}$ & RANK \\
\hline Baseline & 0.7127 & 0.4043 & 0.6251 & 0.4043 & & 0.8556 & 0.4799 & 0.7456 & 0.4799 & \\
\hline OurSystem-Run1 & 0.8427 & 0.5655 & 0.7862 & 0.5475 & 5 & 0.8728 & 0.5945 & 0.8147 & 0.5739 & 9 \\
\hline OurSystem-Run2 & 0.8427 & 0.5179 & 0.7807 & 0.4969 & 8 & 0.8789 & 0.543 & 0.8178 & 0.525 & 15 \\
\hline OurSystem-Run3 & 0.8418 & 0.5541 & 0.7847 & 0.5351 & 7 & 0.8786 & 0.5884 & 0.8193 & 0.5656 & 11 \\
\hline \multirow[t]{3}{*}{ BestSystem } & 0.8429 & 0.6276 & 0.7813 & 0.6095 & 1 & 0.8922 & 0.6867 & 0.8408 & 0.6708 & 1 \\
\hline & \multicolumn{5}{|c|}{ HEADLINES SYS } & \multicolumn{5}{|c|}{ HEADLINES GS } \\
\hline & $\mathrm{F}$ & $+\mathrm{T}$ & $+\mathrm{S}$ & $+\mathrm{TS}$ & RANK & $\mathrm{F}$ & $+\mathrm{T}$ & $+\mathrm{S}$ & $+\mathrm{TS}$ & RANK \\
\hline Baseline & 0.6486 & 0.4379 & 0.5912 & 0.4379 & & 0.8462 & 0.5462 & 0.761 & 0.5461 & \\
\hline OurSystem-Run1 & 0.8078 & 0.5234 & 0.7374 & 0.5099 & 5 & 0.879 & 0.5744 & 0.8096 & 0.5591 & 16 \\
\hline OurSystem-Run2 & 0.7973 & 0.5138 & 0.7369 & 0.5028 & 7 & 0.8859 & 0.5643 & 0.8019 & 0.5554 & 18 \\
\hline OurSystem-Run3 & 0.805 & 0.5185 & 0.7374 & 0.5054 & 6 & 0.8853 & 0.5771 & 0.8089 & 0.562 & 15 \\
\hline \multirow[t]{3}{*}{ BestSystem } & 0.8366 & 0.5605 & 0.7595 & 0.5467 & 1 & 0.8194 & 0.7031 & 0.7865 & 0.696 & 1 \\
\hline & \multicolumn{5}{|c|}{ STUDENT ANSWERS SYS } & \multicolumn{5}{|c|}{ STUDENT ANSWERS GS } \\
\hline & $\mathrm{F}$ & $+\mathrm{T}$ & $+\mathrm{S}$ & $+\mathrm{TS}$ & RANK & $\mathrm{F}$ & $+\mathrm{T}$ & $+\mathrm{S}$ & $+\mathrm{TS}$ & RANK \\
\hline Baseline & 0.6188 & 0.4431 & 0.5702 & 0.4431 & & 0.8203 & 0.5566 & 0.7464 & 0.5566 & \\
\hline OurSystem-Run1 & 0.8162 & 0.5479 & 0.7589 & 0.542 & 3 & 0.8775 & 0.5888 & 0.8102 & 0.5808 & 7 \\
\hline OurSystem-Run2 & 0.8161 & 0.5434 & 0.7481 & 0.5405 & 4 & 0.859 & 0.5758 & 0.791 & 0.5714 & 10 \\
\hline OurSystem-Run3 & 0.8166 & 0.5613 & 0.7574 & 0.5547 & 1 & 0.8505 & 0.5984 & 0.7896 & 0.589 & 6 \\
\hline BestSystem & & & & & & 0.8684 & 0.6511 & 0.8245 & 0.6385 & 1 \\
\hline
\end{tabular}

Table 2: Results for the Baseline, Our System three runs and the Best System for the two subtracks split in the three datasets.

suggest that the system takes advantage of a bigger training set with different domain data. Instead, the size of the mini-batch (that is the difference between run1 and run3) does not seem to have a clear influence on the system performance, since in some cases run1 is higher ranked while in other cases run3 is higher ranked.

Furthermore, Table 2 shows that results for Alignment classification (F) and for Alignment plus STS score $(+\mathrm{S})$ frequently approach the Best System (being the major deficit for $\mathrm{F}$ equal to 0.0393 in Headline SYS for run3 and equal to 0.0349 for $+\mathrm{S}$ in Student Answwer GS dataset for run3) and in a few cases outperform it (e.g. in Headlines GS for F results and in Images SYS for $+S$ results). On the other hand, when also relation type classification is considered (i.e. $+\mathrm{T}$ and $+\mathrm{TS}$ ) we register worse performances, being the minimum difference with the Best System equals to 0.0371 for $+\mathrm{T}$ results and of 0.0368 for +TS results (both in Headlines SYS) and the maximum difference equals to 0.1437 for $+\mathrm{T}$ and to 0.1458 for +TS (both in Images GS). This indicates that type labelling is the hardest subtask for our system, probably because the subtask requires to identify a higher number of classes (i.e. 7 types).

By comparing the rank of the two subtracks SYS and GS, we notice that our system performs much better in the SYS subtrack (being the worst ranking 8 out of 13 for SYS and 18 out of 20 for GS). This fact indicates that our system does not benefit from having already chunked sentence pairs.

Table 3 presents the final rank calculated by considering the mean of +TS results for the three datasets. As previously mentioned, our system performs relatively better when chunk identification is required. Also, it evidences again that run 2 performs worse and that run 1 and run 3 are similar. Overall our system ranked second among 4 systems $(+1$ by the authors) in the SYS subtrack. 


\begin{tabular}{lllll} 
& \multicolumn{2}{c}{ SYS } & \multicolumn{2}{c}{ GS } \\
\cline { 2 - 5 } & \multicolumn{1}{c}{ MEAN } & FANK & \multicolumn{1}{c}{ MEAN } \\
& \multicolumn{1}{c}{ F + TS } & & \multicolumn{1}{c}{ F + TS } & RANK \\
\cline { 2 - 5 } Baseline & 0.428433 & & 0.527533 & \\
Run1 & 0.533133 & 4 & 0.571266 & 11 \\
Run2 & 0.5134 & 6 & 0.5506 & 17 \\
Run3 & 0.531733 & 5 & 0.5722 & 10 \\
BestSystem & 0.552333 & 1 & 0.637733 & 1 \\
\hline
\end{tabular}

Table 3: Mean of the F+TS results in the two subtracks for the Baseline, Our System three runs and the Best System and final rank.

\section{Conclusion and Further Work}

Considering the obtained results, in particular the difference between the runs, we expect our system to be robust also in situation where data from different domains are provided (e.g. training data from several domains and test data on one of them). In fact, for domain adaptation our system seems to require few data of the target domain.

In any case, the system perform better with more training data, independently on the domains involved. As such, further work may include the use of silver data extracted from other datasets, e.g. SICK dataset (Marelli et al., 2014).

In addition, we believe that a deep analysis of the distribution of the type labels and of the STS scores can improve significantly the performance of the system.

Finally, an ablation test can be helpful in identifying the most salient features for the systems, helping to reduce the complexity of the MLP or to develop better topologies.

\section{Acknowledgments}

We are grateful to José G. C. de Souza, Matteo Negri, and Marco Turchi for their suggestions.

\section{References}

Martín Abadi, Ashish Agarwal, Paul Barham, Eugene Brevdo, Zhifeng Chen, Craig Citro, Greg S. Corrado, Andy Davis, Jeffrey Dean, Matthieu Devin, Sanjay Ghemawat, Ian Goodfellow, Andrew Harp, Geoffrey Irving, Michael Isard, Yangqing Jia, Rafal Jozefowicz, Lukasz Kaiser, Manjunath Kudlur, Josh Levenberg, Dan Mané, Rajat Monga, Sherry Moore, Derek
Murray, Chris Olah, Mike Schuster, Jonathon Shlens, Benoit Steiner, Ilya Sutskever, Kunal Talwar, Paul Tucker, Vincent Vanhoucke, Vijay Vasudevan, Fernanda Viégas, Oriol Vinyals, Pete Warden, Martin Wattenberg, Martin Wicke, Yuan Yu, and Xiaoqiang Zheng. 2015. TensorFlow: Large-scale machine learning on heterogeneous systems. Software available from tensorflow.org.

Eneko Agirre, Mona Diab, Daniel Cer, and Aitor Gonzalez-Agirre. 2012. Semeval-2012 task 6: A pilot on semantic textual similarity. In Proceedings of the First Joint Conference on Lexical and Computational Semantics-Volume 1: Proceedings of the main conference and the shared task, and Volume 2: Proceedings of the Sixth International Workshop on Semantic Evaluation, pages 385-393. Association for Computational Linguistics.

Eneko Agirre, Daniel Cer, Mona Diab, Aitor GonzalezAgirre, and Weiwei Guo. 2013. sem 2013 shared task: Semantic Textual Similarity, including a Pilot on Typed-Similarity. In In* SEM 2013: The Second Joint Conference on Lexical and Computational Semantics. Association for Computational Linguistics. Citeseer.

Eneko Agirre, Carmen Banea, Claire Cardie, Daniel Cer, Mona Diab, Aitor Gonzalez-Agirre, Weiwei Guo, Rada Mihalcea, German Rigau, and Janyce Wiebe. 2014. Semeval-2014 task 10: Multilingual Semantic Textual Similarity. In Proceedings of the 8th International Workshop on Semantic Evaluation (SemEval 2014), pages 81-91.

Eneko Agirre, Carmen Baneab, Claire Cardiec, Daniel Cerd, Mona Diabe, Aitor Gonzalez-Agirrea, Weiwei Guof, Inigo Lopez-Gazpioa, Montse Maritxalara, Rada Mihalceab, et al. 2015. Semeval-2015 task 2: Semantic Textual Similarity, English, Spanish and Pilot on Interpretability. In Proceedings of the 9th International Workshop on Semantic Evaluation (SemEval 2015), pages 252-263.

Eneko Agirre, Aitor Gonzalez-Agirre, Iñigo LopezGazpio, Montse Maritxalar, German Rigau, and Larraitz Uria. 2016. Semeval-2016 task 2: Interpretable semantic textual similarity. In Proceedings of the 10th International Workshop on Semantic Evaluation (SemEval 2016), San Diego, California, June.

Walter Daelemans and Antal Van den Bosch. 2005. Memory-based language processing. Cambridge University Press.

Tom De Smedt and Walter Daelemans. 2012. Pattern for python. The Journal of Machine Learning Research, 13(1):2063-2067.

Christiane Fellbaum. 1998. WordNet. Wiley Online Library.

Diederik Kingma and Jimmy Ba. 2014. Adam: A 
method for stochastic optimization. arXiv preprint arXiv:1412.6980.

Marco Marelli, Stefano Menini, Marco Baroni, Luisa Bentivogli, Raffaella Bernardi, and Roberto Zamparelli. 2014. A sick cure for the evaluation of compositional distributional semantic models. In Proceeding of Language Resources and Evaluation Conference (LREC 2014), pages 216-223.

I. Dan Melamed. 1998. Manual annotation of translational equivalence: The blinker project. Technical Report 98-07, Institute for Research in Cognitive Science, Philadelphia.

Tomas Mikolov, Ilya Sutskever, Kai Chen, Greg S Corrado, and Jeff Dean. 2013. Distributed representations of words and phrases and their compositionality. In Advances in neural information processing systems, pages 3111-3119.

Jeffrey Pennington, Richard Socher, and Christopher D Manning. 2014. Glove: Global vectors for word representation. In Proceedings of Empirical Methods in Natural Language Processing (EMNLP 2014), pages 1532-1543. 